\title{
Dynamic fracture of a dual phase automotive steel
}

\author{
Sarath Chandran ${ }^{1 *}$, Patricia Verleysen ${ }^{1}$, Junhe Lian², Wenqi Liu ${ }^{2}$, Sebastian Münstermann ${ }^{2}$ \\ ${ }^{1}$ DyMa Lab, EEMMeCS department, Ghent University, Technologie park 903, Zwijnaarde 9052, Ghent, Belgium \\ ${ }^{2}$ Steel Institute, RWTH Aachen University, Intzestraße 1, 52072 Aachen, Germany
}

\begin{abstract}
Dynamic testing of sheet metals has become more important due to the need for more reliable vehicle crashworthiness assessments in the automotive industry. The study presents a comprehensive set of experimental results that covers a wide range of stress states on a dual phase automotive sheet steel. Split Hopkinson bar tensile (SHBT) tests are performed on dogbone shaped samples to obtain the plastic hardening properties at high strain rates. A set of purpose designed sample geometries comprising of three notched dogbone tension samples is tested at high strain rates to characterise the dynamic damage and fracture properties under well controlled stress states. The geometry of the samples is optimised with the aid of finite element analysis. During the tests, high speed photography together with digital image correlation are implemented to acquire full field measurements and to gain more insight into the localisation of strains at high strain rates. An experimental-numerical approach is proposed to effectively determine the fracture characteristics of the dual phase steel under extreme conditions. A modified Bai-Wierzbicki model is implemented to assess the damage initiation and subsequent failure. Additionally, the fracture mechanisms are studied utilizing scanning electron microscopy.
\end{abstract}

\section{Introduction}

The automotive industry is constantly evolving and thus it is necessary to build research, development and innovation capacity. An improved understanding of the behavior of automotive materials at high velocity is driven by the challenges of diverse crash legislation and competition amongst car makers. The strength of a sheet steel product is dependent on the speed at which it is deformed $[1,2]$. The mechanical behavior of materials under dynamic or impact loading is different from that under static loading. When a structure deforms dynamically, the inertia effect and the propagation of stress waves are so important that the material properties are influenced by the strain rate [35]. Tensile testing of metallic sheet materials at high strain rates is important to achieve a reliable analysis of vehicle crashworthiness. In order to investigate the material component performance of crash boxes, it is essential to characterize the material mechanical properties, including the elastic-plastic deformation and damage/ fracture properties at dynamic rates.

An extensive experimental program at lab scale is designed involving dogbone and notched tension specimens to cover a wide range of stress states. These tests are performed at quasi static to high speed conditions to obtain the plasticity and fracture description of the material response at various strain rates.

\section{Materials and methods}

\subsection{Material}

The investigated material is an automotive dual phase steel (DP-K1000) provided by ThyssenKrupp as sheets of thickness $1.5 \mathrm{~mm}$. The microstructure consists of equal proportions of ferrite and martensite with a grain size less than $2 \mu \mathrm{m}$. All the tensile samples are extracted in the rolling direction. The regular dimensions are obtained using waterjet cutting and electro discharge machining $(\mathrm{EDM})$ is employed for the notches.

\subsection{Methods}

\subsubsection{Static tests}

An Instron material testing machine (Model 5569) is used for the low strain rate experiments. Similar boundary conditions are ensured for both static and dynamic tests. Tests are performed at three different crosshead velocities, aiming at strain rates of $0.0001,0.001$ and $0.01 \mathrm{~s}^{-1}$ in the central gauge or notch section of the samples. Digital image correlation (DIC) is used to obtain the local strain from a speckle pattern applied to the sample surface recorded during the deformation. Three LVDTs attached to the loading bars are also used to measure the sample elongation. The force is measured using a load cell with a capacity of $50 \mathrm{kN}$.

\subsubsection{Dynamic tests}

The high strain rate tests are carried out on split Hopkinson tensile setup. Split Hopkinson bars are widely used to 
study the constitutive, damage and fracture behavior of materials at strain rates from $100 \mathrm{~s}^{-1}$ up to $10000 \mathrm{~s}^{-1}$. One of the main characteristics of Hopkinson experiments, in comparison with other high strain rate test techniques, is that a high strain rate deformation is imposed while the sample is in dynamic equilibrium. Additionally, the determination of the force on the specimen is done without the use of a load cell and the measurement of the specimen elongation is made without directly monitoring the specimen length. The concept of the split Hopkinson bar setup involves a test sample sandwiched between two long bars, an input and output bar. For tensile tests, most often, a tube-like impactor is put around the input bar and is accelerated towards an anvil at the outer end of the input bar. Thus a tensile wave, the so-called incident wave, is generated and propagates along the input bar towards the specimen. The strain rate in the sample can be adjusted by the velocity of the impactor.

The incident wave interacts with the specimen, generating a reflected wave and a transmitted wave. The incident, reflected and transmitted wave are measured, usually by means of strain gages, at well-chosen locations on the Hopkinson bars. From these waves, the total force and elongation history of the specimen can be determined based on the principle of one dimensional elastic wave propagation in slender bars [6]. A local strain measurement is used based on high speed camera imaging and DIC to monitor non-homogenous strain fields and to compensate for the overestimation of the strain from strain gauges especially for dog bone shaped tensile samples [7]. The DyMaLab SHTB setup has a total length of $11 \mathrm{~m}$ and allows loading times upto 1.2 milliseconds.

\subsubsection{Finite element (FE) modelling}

To come to an in-depth understanding of the experimental results, finite element models have been built of the test configurations using the commercial program Abaqus explicit. As the mesh is a key factor influencing the FE simulations, especially damage mechanics simulations in this study, consistent element type and size at the critical plastic deformation zone across all models are employed. Fine and regular 3D solid brick elements with reduced integration (C3D8R) are used. The critical element size is $0.1 \mathrm{~mm}$. To model the material, J2 plasticity combined with a user material subroutine (VUMAT) for damage and fracture is adopted. Boundary conditions similar to those in the actual tests are implemented. The velocity is imposed on one end of the specimen.

\section{Test specifications}

In the test program, a wide range of strain rates and stress states is considered to assess the strain rate sensitivity and damage properties of the material. Both quasi static and dynamic tests are performed at three different strain rates for each sample geometry. Two tests are performed for each test condition.
The general shape of the tensile specimen is a typical dogbone. The dogbone specimen used to characterise the strain rate dependent plastic hardening of the DP1000 steel, features a parallel gauge section with a length of 6 $\mathrm{mm}$ and width of $3 \mathrm{~mm}$, see Fig. 1a. The radius of transition between the parallel gauge section and grip section is $1 \mathrm{~mm}$. Notched specimens are used to assess the material damage and fracture properties. The notches give rise to non-axial stresses and thus higher stress triaxialities. Purpose based design with notched radii of $0.4,1.8$ and $4.5 \mathrm{~mm}$ are employed to target specific range of triaxialities, see Figs. $1 \mathrm{~d}, \mathrm{c}$ and b. Readers are directed to [8] for detailed analysis on the optimisation of the geometry design. Correspondingly, the minimum width of the notch sections are $1.6,2$ and $1.6 \mathrm{~mm}$ respectively.

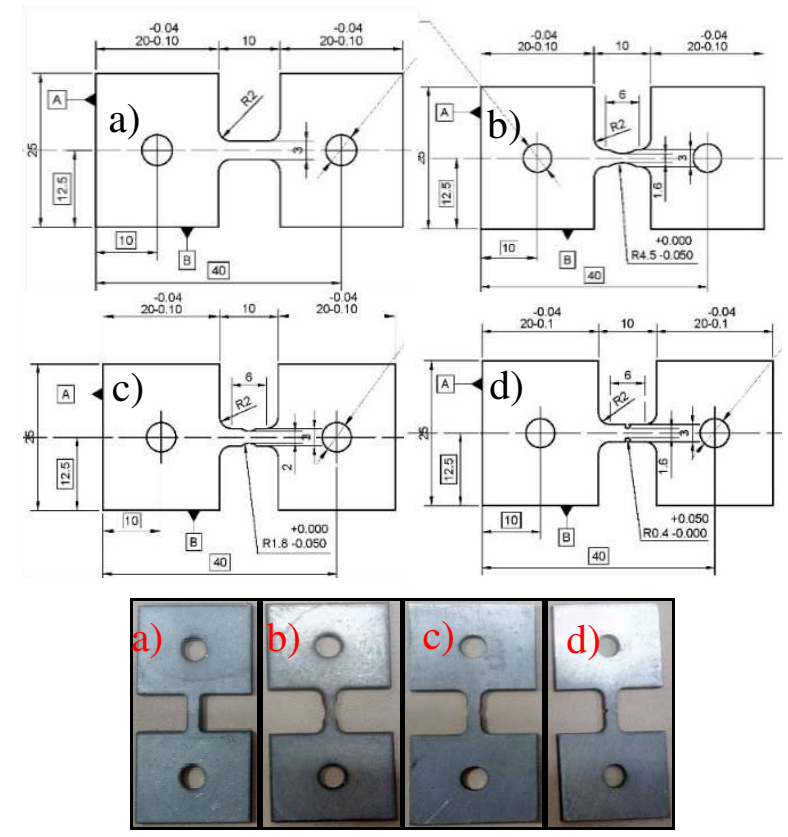

Fig. 1. a) Regular dogbone b) R4.5 c) R1.8 d) R0.4.

\section{Results and discussion}

\subsection{Uniaxial tensile test}

Tensile tests carried out on the dogbone shaped specimen allow obtaining the plastic hardening characteristics. Strain rates of $0.0001,0.001$ and $0.01 \mathrm{~s}^{-1}$ are imposed under static testing whereas under dynamic conditions, averaged strain rates (during plastic deformation) of 490 , 710 and $980 \mathrm{~s}^{-1}$ are obtained.

Fig. 2 shows the representative engineering stress-strain curves for the dual phase steel at strain rates ranging from the quasi static to dynamic regime. Adiabatic heating conditions under high strain rates result in a localised temperature increase which diminishes the rate effect on flow stress subsequently leading to depreciated sensitivity under dynamic rates. Positive effect of strain rate is observed for both ultimate stress and absorbed energy per unit volume, see Fig.3. Lower fracture strain is obtained for dynamic tests.

\subsection{Specimen geometries}




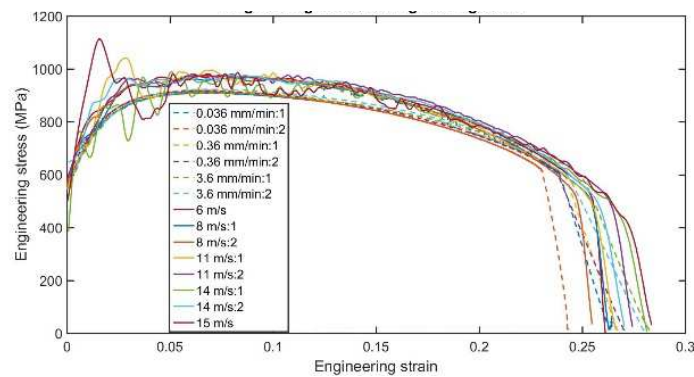

Fig. 2. Quasi static and dynamic engineering stress-strain curve for a uniaxial tensile test.

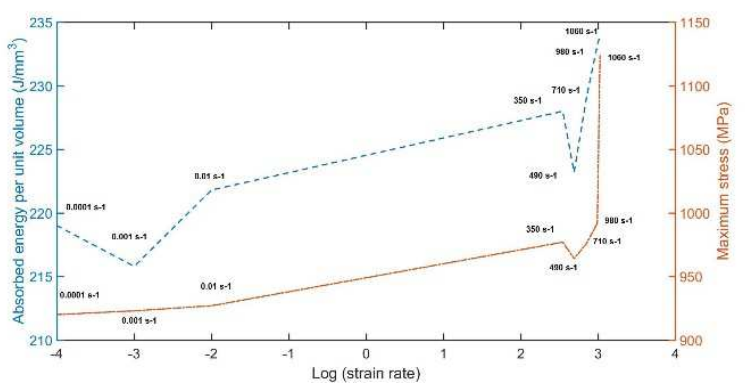

Fig. 3. Effect of strain rate from a uniaxial tensile test.

\subsection{Fracture tensile tests}

Figs.4 present the static and dynamic engineering stressdisplacement curves for all the notched tensile specimens. Engineering stress is the average tensile stress in the notch (force / initial area of notch section). Higher stress levels are observed in comparison with the dogbone shaped specimens owing to the multi axial stress state in the notches. This effect amplifies with increasing notch sharpness. Plastic hardening and damage/fracture behaviour under featured stress states are extracted from the tests on notched specimens.

Similar to the uniaxial tensile test, the strain rate influence is considerable during the transition from static to dynamic deformation rates for notched samples. Deformation levels are substantially reduced with the severity of the notches due to the higher stress triaxialities with increasing notch sharpness. Moreover, non axial stresses directly translates to a deteriorating effect on the material ductilty. Fracture strain measurements, digital image correlation and finite element simulations confirms this obervation. Fig. 5 shows the phenomenon of strain localisation for all the tensile specimen geometries. In Fig 5 , corresponding axial strains for dogbone, R0.4, R1.8 and R4.5 specimensare $0.29, \quad 0.068,0.149$ and 0.255 respectively.

\subsection{Effect of stress state}

Notched tensile geometries are designed to cover discrete range of stress state between uniaxial and plane strain tension. Sample dimensions in Fig.1 give rise to initial triaxialities of $0.33,0.38,0.41$ and 0.52 respectively for dogbone, R4.5, R1.8 and R0.4 tensile specimen geometries. Inhomogeneous stress and strain distribution are commonly observed in the gauge or notch section.
Finite element simulations, see Fig.6are employed to describe the evolution and distribution of triaxiality.
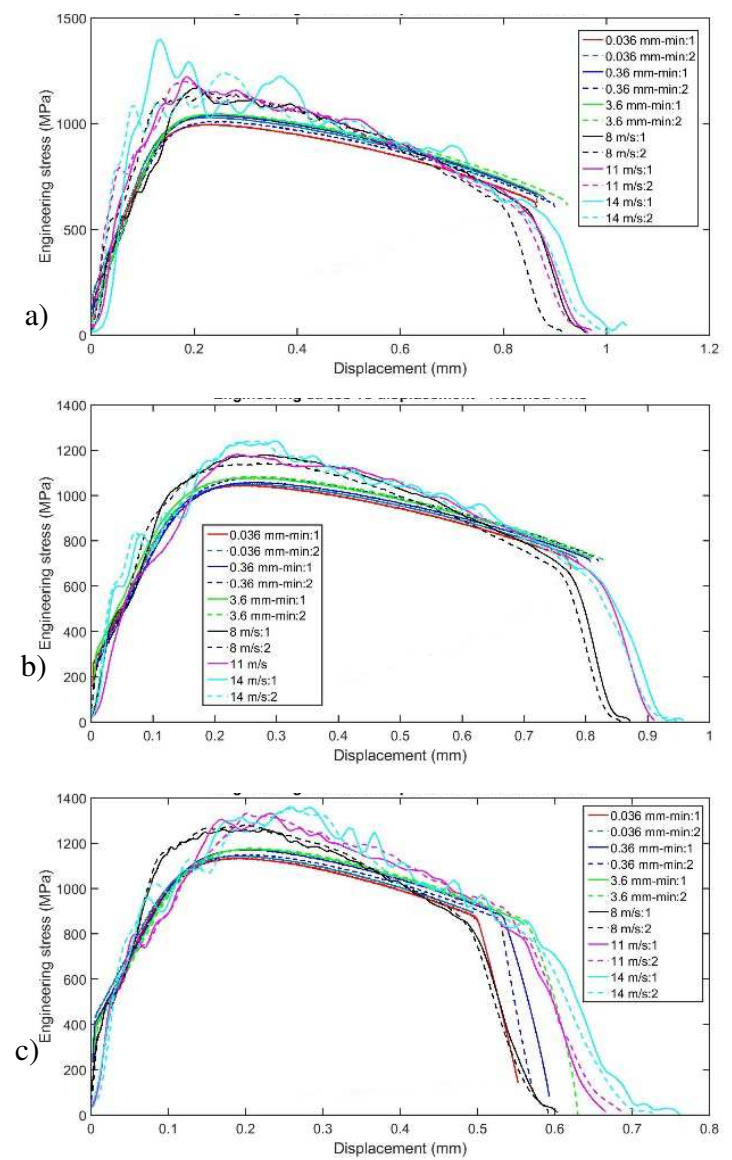

Fig. 4. Quasi static and dynamic engineering stress-displacement curves for notched specimens with radii a) R4.5 b)R 1.8 and c) R0.4.

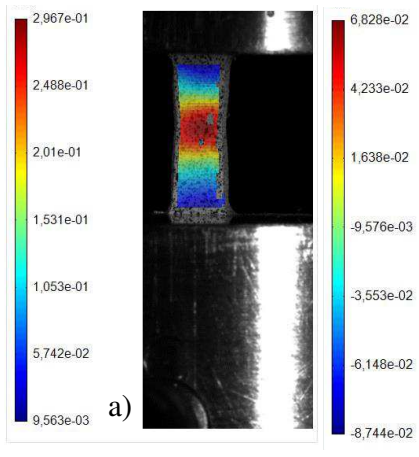

b)
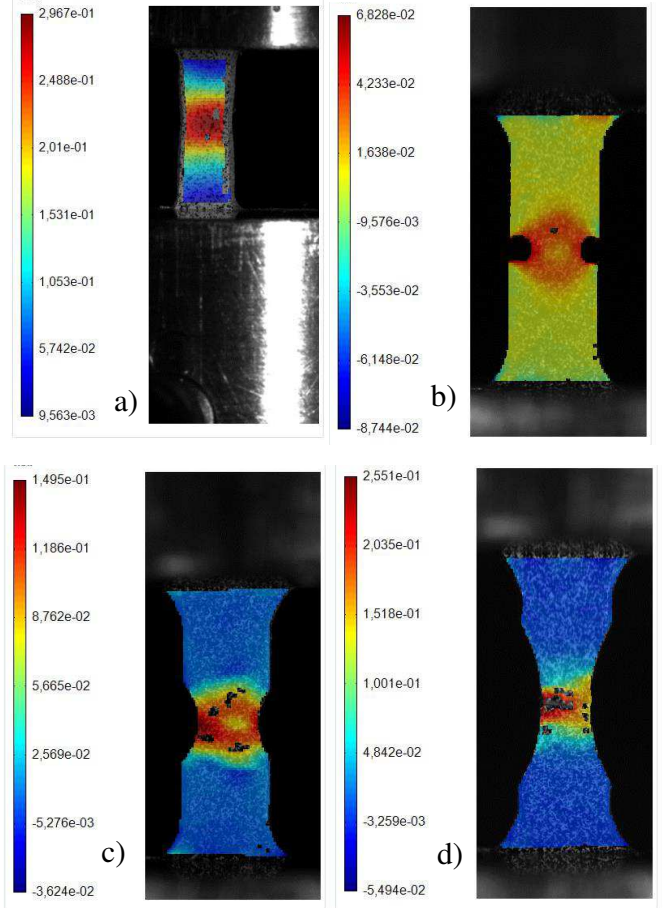

Fig. 5. Digital image correlation for tensile specimens a) Uniaxial b) R0.4 c) R1.8 and d) R4.5. 


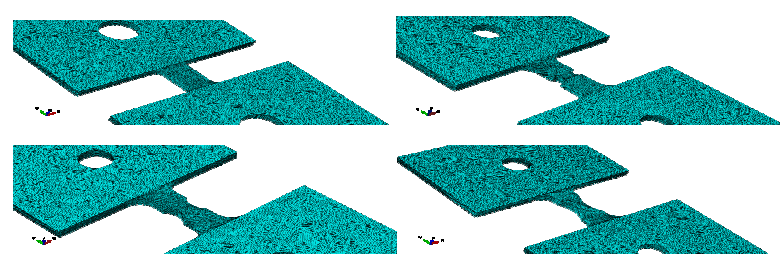

Fig. 6. Finite element model of all the tensile specimens.

Stress state is predominantly defined by both stress triaxiality and Lode angle parameter and knowledge of their evolution is paramount for quantifying damage.

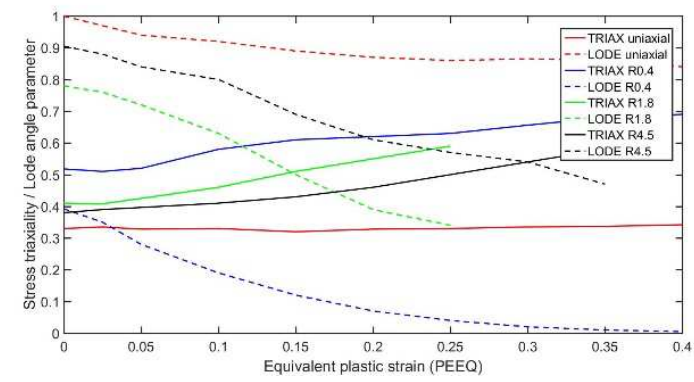

Fig. 7 Evolution of stress triaxiality and Lode parameter with respect to equivalent plastic strain.

Evolution of stress triaxiality and Lode angle parameter in the central element for all the tensile specimens are represented as a function of Mises plastic equivalent strain (PEEQ). Triaxiality increases during deformation. On the contrary, a decreasing trend is observed for Lode angle parameter due to the development of non axial stresses.

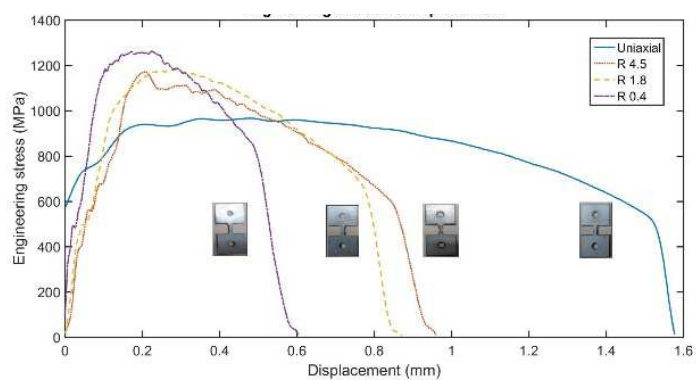

Fig. 8 Stress-displacement curve for all the tensile specimens.

As previously mentioned, featured stress state has a detrimental effect on the deformation capacity. Fig 8 presents stress-displacement curves for all the tensile specimen geometries at a strain rate of $490 \mathrm{~s}^{-1}$. Since, different volumes of the material are involved, further confirmation of the degrading effect of triaxiality on ductility is executed using finite element simulations and final strain measurements from the fracture surface.

\subsection{Effect of strain rate}

Strain rate sensitivity of the material is demonstrated in Fig 9, wherein tensile strength is presented as a function of the logarithm of strain rate. Both static and dynamic tests are considered on all the tensile specimens. The strain rate has a positive influence on the maximum tensile strength, more so in the dynamic regime. This effect increases with increase in triaxiality. The strain rate also has a clear impact on the energy absorbed by the material, as can be seen in Fig. 10 where absorbed energy is plotted as a function of strain rate for all the specimens. Varying dependencies are observed in the static regime however, in the dynamic regime, absorbed energy consistently increases with strain rate.

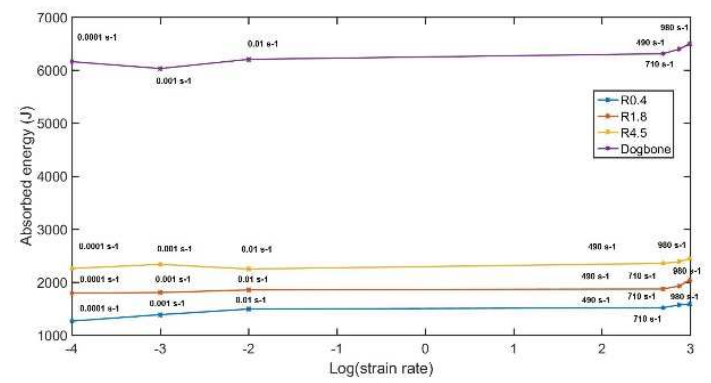

Fig. 9. Effect of strain rate on the ultimate stress.

The effect of strain rate is most pronounced for regular dogbone specimen in the dynamic regime.

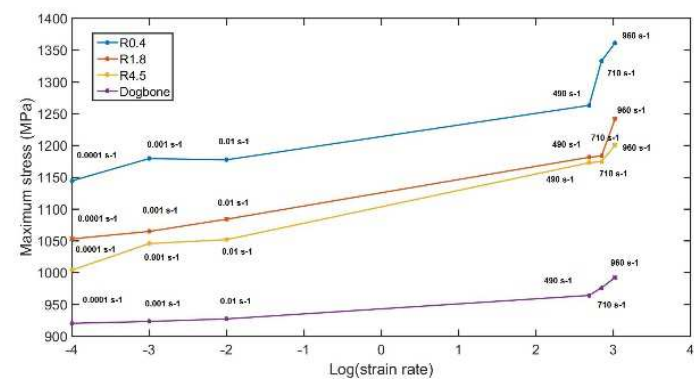

Fig. 10. Effect of strain rate on absorbed energy.

\subsection{Fracture strain}

Fracture strain is one of the most interesting fracture properties, since it quantifies the material deformation capacity unambiguously. For a comprehensive assessment of the influence of the stress state on ductility, post mortem fracture surfaces have been observed. Fully ductile characteristics are observed from the fracture surface for the dogbone specimen. Fig. 11a exhibits the fracture surface of a specimen after uniaxial tensile test at a strain rate of $450 \mathrm{~s}^{-1}$. Large proportions of dimples and smaller voids indicate completely ductile failure for this test sample and condition. Average values of the fracture strain are measured from the fracture surface for all the sample geometries under all the test conditions.

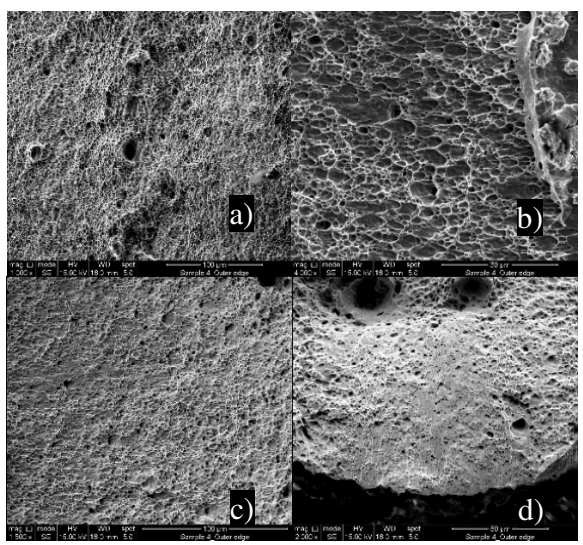

Fig. 11. Fracture surfaces of all the tensile specimens a) Uniaxial tensile b) R4.5 c) R1.8 and d) R0.4. 
Measurement of the fracture strain on the fracture surface showed that stress state, indeed has a negative influence on the material response in terms of deformation capacity, see Fig.12, representing the averaged fracture strains for each stress state. However, marginally lower values for fracture strains are obtained for dynamic tests which shows that higher energy absorption at high strain rates is primarily and dominantly contributed by the positively affected flow stresses. Fracture surfaces of R4.5, R1.8 and R0.4 specimens are displayed in Figs. 11.b,c and $\mathrm{d}$ respectively.

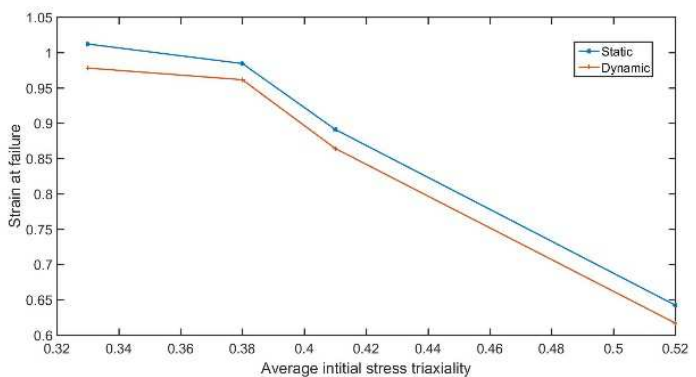

Fig. 12. Fracture strain vs Stress triaxiality.

Although ductile fracture is observed for R4.5 sample, larger dimples and lesser voids indicate lesser nucleation and coalescence sequence thereby explaining lower deformation for this geometry. Elongated dimples with a certain orientation in the transverse plane indicate shear and cleavage zones for R0.4 and R1.8. Cleavage facets further contributed to early fracture and lower strains for these geometries.

\subsection{Modified Bai-Wierzbicki (MBW) Model}

The original Bai-Wierzbicki model addresses the influence of stress triaxiality and Lode angle parameter on the plasticity and fracture behaviour of metals. Both the yield stress $\sigma y l d$, and fracture strain $\overline{\varepsilon_{p}} f$ are formulated with respect to the two parameters. The motivation of the MBW model is to properly describe the non-negligible impact of damage on strength and ductility and dependence of stress state on the accumulation [9-10]. The hybrid MBW damage plasticity model is formulated with three main constituents:

(1) A plasticity model to characterize the material behavior before damage initiation.

(2) A phenomenological criterion to indicate the initiation of damage.

(3) A damaged-induced softening part to characterize the post-damage material behavior.

The yield potential of the MBW model reads: $\varphi_{M B W}=\sigma_{e}-(1-D) \cdot \sigma_{y}\left(\varepsilon^{\bar{p}}, \dot{\varepsilon} \bar{p}\right) \cdot f(T) \cdot f(\overline{\theta)} \leq 0$ (1) where $\sigma_{e}$ is the equivalent stress; $\sigma_{y}\left(\varepsilon^{\bar{p}}, \dot{\varepsilon} \bar{p}\right)$ is the yield stress :

$\sigma_{y}\left(\varepsilon^{\bar{p}}, \dot{\varepsilon \bar{p}}\right)=\sigma_{y}\left(\varepsilon^{\bar{p}}\right) \cdot\left(1+\mathrm{c}_{1} \dot{\varepsilon} \ln \varepsilon^{p} / \dot{\varepsilon_{0}}\right)+\mathrm{c}_{2}^{\dot{\bar{\varepsilon}}} \cdot \overline{\sigma_{0}}\left(\varepsilon^{p /} / \overline{\varepsilon_{0}}-1\right)$

which accounts for the strain hardening and the influence of strain rate, $f(T)$ and $f(\bar{\theta})$ are the temperature and stress state (Lode angle parameter) effects on the yield stress respectively.

$$
f(T)=c_{1}^{T} \cdot \exp \left(c_{2}^{T} \cdot T\right)+c_{3}^{T}
$$

$$
\begin{gathered}
f(\bar{\theta})=\left[c_{\theta}^{s}+\left(c_{\theta}{ }^{a x}-c_{\theta}^{s}\right) \cdot\left(\omega-\omega^{m+1} / m+1\right)\right] \\
\operatorname{avec} \omega=\left[\sqrt{ } 3 /(2-\sqrt{ } 3) \mid \sec \left(\theta \cdot \prod / 6\right)-1\right]
\end{gathered}
$$

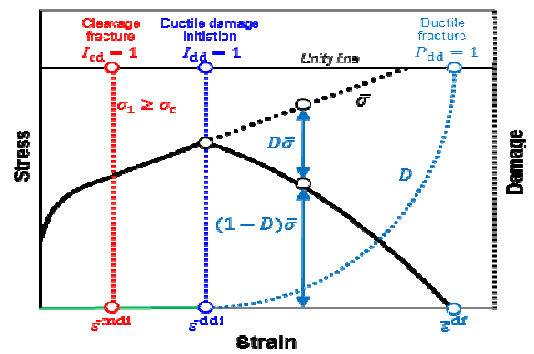

Fig. 13. Representation of the MBW model.

$\mathrm{D}$ is the damage variable. The damage induced softening effect is coupled to the yield potential with the factor (1-D). The evolution of the damage variable $D$ is controlled by a damage initiation locus and a subsequent energy based evolution law. It is equal to zero before the damage initiation. The damage initiation strain, $\overline{\varepsilon p}$, of the MBW model is defined as a function of stress triaxiality and Lode angle parameter, for cleavage behaviour $\varepsilon^{m c i}$ and for ductile behaviour, $\varepsilon^{d d i}$. Four material parameters, $\mathrm{C}_{1}-\mathrm{C}_{4}$ for cleavage behaviour and $\mathrm{D}_{1}-\mathrm{D}_{4}$ for ductile behaviour needs to be calibrated to define the damage initiation locus (DIL) according to the following equations:

$$
\begin{aligned}
& \varepsilon^{-}{ }^{-} c i\left(\eta_{\text {avg }}, \bar{\theta}_{\text {avg }}\right)=\left[C_{1} e^{-C 2} \eta-C_{3} e^{-C 4} \eta\right] \bar{\theta}^{-2}+C_{3} e^{-C 4} \eta \\
& \varepsilon^{d} \text { di }\left(\eta_{\text {avg }}, \bar{\theta}_{\text {avg }}\right)=\left\{\quad+\infty, \text { for } \eta \leq \eta_{c}\right. \\
& \left.\left[D_{1} e^{-D}{ }_{2}^{\eta}-D_{3} e^{-{ }^{D}} \eta\right] \vec{\theta}+D_{3} e^{-D 4} \eta \text {, for } \eta>\eta_{c}\right\}
\end{aligned}
$$

where $\eta_{\mathrm{c}}$ is the cut off value [11], normally equal to $1 / 3$ for metals, under which failure suppresses during plastic deformation. Based on the energy point of view, tow indicators are defined for both cleavage fracture initiation, $\mathrm{I}_{\mathrm{cf}}$, and ductile damage initiation, $\mathrm{I}_{\mathrm{dd}}$, according to the following equations:

$$
\begin{gathered}
I_{c f}=\int_{0}^{\varepsilon \bar{p}} d \varepsilon^{\bar{p}} / \varepsilon^{m^{-} c i}\left(\eta_{\text {avg }}, \bar{\theta} \text { avg }\right) \\
I_{d d}=\int_{0}^{\bar{p}} d \varepsilon^{\bar{p}} / \varepsilon^{\bar{d}} d i\left(\eta_{\text {avg }}, \bar{\theta}_{\text {avg }}\right)
\end{gathered}
$$

where $\varepsilon^{p}$ is the equivalent plastic strain to fracture. An energy based evolution rule is proposed to describe the damage propagation in this investigation. By defining an energy dissipation parameter $G f$, and the critical damage value $D_{c r}$ as the material parameters, the ductile damage propagation rule is :

$$
P d d=\int_{s c \pi}^{\varepsilon \bar{p}}\left(\sigma_{y i} / G f\right) / D_{c r}\left(\eta_{a v g}, \bar{\theta}_{a v g}\right) d \varepsilon^{\bar{p}}
$$

For non-proportional loading, the characteristic strain $\overline{\varepsilon_{c}} d d i$ at the ductile damage initiation is equal to $\overline{\varepsilon p}$ at $\mathrm{I}_{\mathrm{dd}}=1$. $\mathrm{G}_{\mathrm{f}}$ controls the rate of damage accumulation and the softening rate. The material is assumed to fail when $\mathrm{D}=\mathrm{D}_{\mathrm{cr}}$; $\mathrm{D}_{\mathrm{cr}}$ can be set to be stress state dependent $[9,12]$.

$$
D_{c r}\left(\eta_{\text {avg }}, \bar{\theta}_{\text {avg }}\right)=\left[E_{1} e^{-E 2} \eta_{-} E_{3} e^{-E 4} \eta\right] \quad \vec{\theta}^{2}+E_{3} e^{-E 4} \eta
$$

Depending on the loading process, $\mathrm{D}$ is consequently distributed in the following range:

$$
\begin{array}{ll}
\{0 & \text { for } I_{c f}<1^{\wedge} I_{d d}<1 \\
0 & \text { for } I_{c f} \geq I^{\wedge} \sigma 1<\sigma c^{\wedge} I_{d d}<1 \\
1 & \text { for } I c f \geq 1^{\wedge} \sigma 1 \geq \sigma c^{\wedge} I_{d d}<1 \\
D=\quad & \text { for Idd } \geq 1^{\wedge} P d d<1
\end{array}
$$




$$
\left.1 \quad \text { for } I d d \geq 1^{\wedge} P d d \geq 1 \quad\right\}
$$

The combined Swift-Voce law is employed for the flow curve derivation based on the quasistatic, room temperature tensile test, with an extension to $\varepsilon=3$. The equations are listed in following, and the flow curve fitting is presented in Fig 14.

Swift power law:

$$
\overline{\sigma y}(\overline{\varepsilon p})=A(\bar{\varepsilon} \overline{0}+\overline{\varepsilon p})^{n}
$$

Voce exponential law:

$$
\overline{\sigma^{V}}(\overline{\varepsilon p})=k_{0}+Q\left(1-e^{-\beta d^{p}}\right)
$$

Combined Swift and Voce:

$$
\overline{\sigma^{S}-V}(\overline{\varepsilon p})=\alpha \cdot \overrightarrow{\sigma_{y}}(\overline{\varepsilon p})+(1-\alpha) \cdot \overrightarrow{\sigma_{y}}(\overline{\varepsilon p})
$$

Hardening and strain rate sensitivity parameters are thereby calibrated based on the results from the uniaxial tensile tests. Temperature sensitivity parameters are excluded from the present study. As mentioned before, calibrated parameters are given as input to the model via a user material subroutine (VUMAT). Von Mises plasticity model is employed with the experiments to derive the damage initiation locus. Damage initiation point is quantified based on the instant at which deviation of simulated force from the experiment exceeds $1 \%$. Correspondingly, the element with the maximum equivalent plastic strain is identified as the critical element. For all the investigated samples, damage initiation point happens after the attainment of the maximum force. Vertical lines in Fig 16 represent the damage initiation point for the respective specimen geometries.

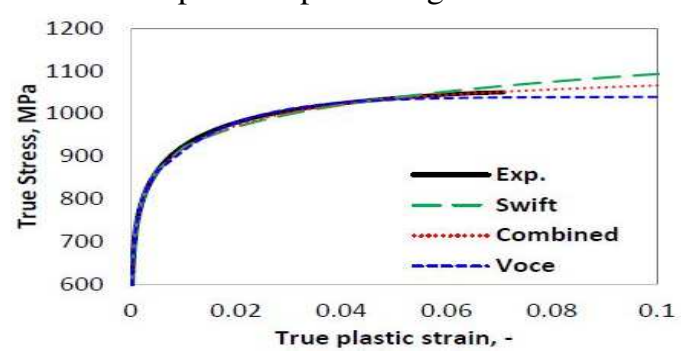

Fig. 14. Experimental and extrapolated flow curve from combined Swift-Voce.

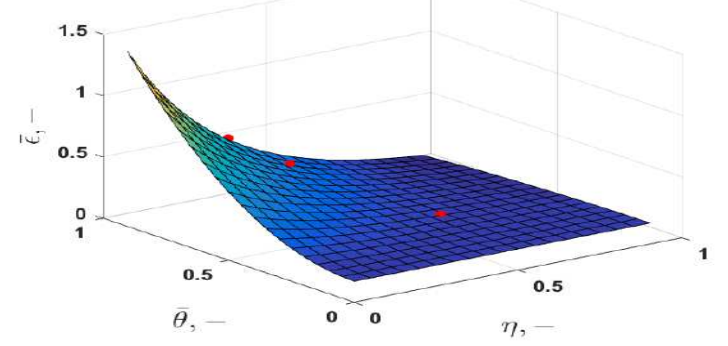

Fig. 15. Fitting of damage initiation locus.

Earlier onset of damage is observed under dynamic conditions thereby explaining lower fracture strain. Furthermore, increase in triaxiality lead to earlier damage and fracture appearance for the dual phase steel. Damage initiation is thus fitted based on the values of stress triaxiality, Lode angle parameter and equivalent plastic strain at the damage initiation point, see Fig. 15. Damage induced softening parameters; $\mathrm{G}_{\mathrm{f}}$ and $\mathrm{D}_{\mathrm{cr}}$ are kept constant $\left(\mathrm{G}_{\mathrm{f}}=2500, \mathrm{D}_{\mathrm{cr}}=0.22\right)$ in the present scenario for study on damage propagation and determination of damage parameters.

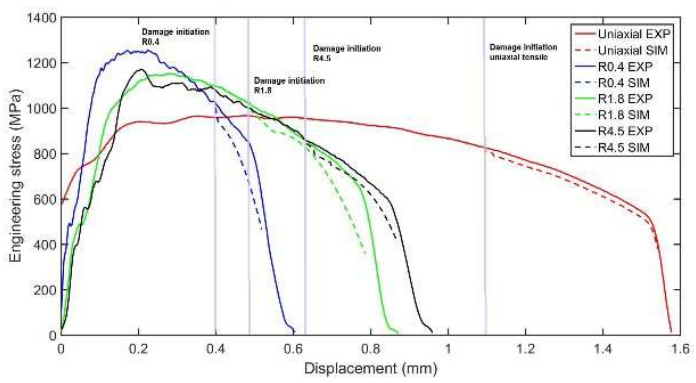

Fig. 16. Experimental and simulated stress- displacement curves.

Fig. 16 shows the calibration results. Larger deviations are observed at higher stress states thereby necessitating the need for the study of stress state influence on damage induced softening parameters for better correspondence.

\section{Conclusion}

The mechanical properties of DP1000 steel have been investigated on lab scale by means of uniaxial and fracture tensile tests. The stress state and strain rate effects on the plasticity and fracture behaviour of DP-K1000 have been analysed. Following conclusions were derived from the evaluation process:

- The material combines high strength with reasonable ductility.

- A ductile fracture is mostly observed for all the specimens at all the strain rates.

- Influence of strain rate is predominant in the transition from static to dynamic range of testing and higher strain rate will lead to higher strength and earlier damage and fracture occurrence at lower deformation levels.

- Decrease in notch radius results in decreased Lode angle parameter and increased stress triaxiality, and further leads to higher axial stresses and earlier damage and fracture appearance.

The author would like to acknowledge the support of Research Fund for Coal and Steel (RFCS) of the European Commission and the partners of the 'Toolkit' project.

\section{References}

1. H. Huh, J. H. Lim, Int J. Auto Tech, 10, 2, 195-204 (2009)

2. A.Niechajowicz, A.Tobota, ACME, 8, 129-137 (2008)

3. J. Slota, E. Spišák: Metalurgija, 47, 1, 13-17 (2008)

4. E. Čižmárová et al.Chemical Let., 105 , 16, 546-548 (2011)

5. H. Huh et al., Int. Journal of Mech. Scien., 50 , 918931 (2008)

6. H. Kolsky, Proc Phys Soc London , B 62 ,676-700 (1949)

7. P. Verleysen et.al , Int J Imp Eng 30, 239-253 (2004)

8. S. Chandran et.al; ProEng , 197, pp.204-2013 (2017)

9. B. Wu et.al.; FFEMS (2017)

10. J. Lian et.al; IJDM 22: 188-218 (2012)

11. Y.L.Bai et.al; Int.J.Plast. 24: 1071-1096 (2008)

12. J. Lian et al.; IJDM 24 (3) 383-417 (2015) 